\title{
Anomalies in Pendulum Behaviour Observed During Conjunctions Mercury/Sun on 11 November 2006, 14 July 2009, and 17 November 2015
}

\author{
Dimitrie Olenici \\ Astronomical Observatory Department, Stefan cel Mare University, Suceava, Romania \\ Email: dimitrieolenici@hotmail.com
}

\begin{abstract}
During the Mercury transit on 11 November 2006, the inferior conjunction of Mercury on 14 July 2009, and the occultation of Mercury on 17 November 2015, a series of experiments were carried out to check for anomalous pendulum behaviour. The results confirm the appearance of anomalous variations in the speed of precession of the plane of oscillation of the pendulum (the socalled Allais effect), anomalous variations of the period of oscillation of the pendulum (the so called Jeverdan-Rusu-Antonescu effect), anomalous variations of the proportion between the major and minor semi axes of the ellipse of oscillation of the pendulum, and anomalies in the chirality of revolution of the pendulum around its ellipse of oscillation.
\end{abstract}

Keywords: Paraconical, pendulum, syzygy, eclipse, conjunction, opposition, torsind, Allais, effect, precession.

\section{Introduction}

It is well known that, during the solar eclipses of 30 June 1954 and 2 October 1959, Maurice Allais (a Nobel laureate in 1987) observed a significant perturbation in the precession of the plane of oscillation of a pendulum [1].

Nowadays this perturbation is called the "eclipse effect" or the "Allais effect".

Also, during the total solar eclipse of 15 February 1961, Gheorghe Jeverdan, Gheorghe Rusu, and Virgil Antonescu discovered that the period of a pendulum was affected [2]. Nowadays this perturbation is known as the "Jeverdan-Rusu-Antonescu eclipse effect".

Starting with the total solar eclipse of 11 August 1999, we have repeated many such experiments, and have found that similar perturbations also appear during lunar eclipses, conjunctions of planets with the Sun, conjunctions between planets, and oppositions and transits of planets - in fact, generally during syzygies. Also we have found that the proportion between the semi-axes of the ellipse of oscillation of the pendulum and the sense of revolution of the pendulum bob around the ellipse of oscillation, i.e. the chirality, are affected during syzygy events, even if those events are not optically visible in the observational location.

For these reasons we have proposed that all these perturbations should be termed "syzygy effects" [3]. In this article we present results of this type obtained during three conjunctions of the planet Mercury with the Sun, as follows: the Mercury transit of 11 November 2006, the inferior conjunction of 15 July 2009, and the occultation of 17 November 2015.

\section{Pendulums and Methodology}

In our experiments we have always utilized aerodynamic paraconical pendulums. The paraconical pendulum was invented by Maurice Allais, and is a pendulum suspended upon a ball that is capable of rolling in any direction upon a horizontal flat plate. The bob of Allais's pendulum was, in most cases, a vertical disc [1]. However, in order to reduce air friction, aerodynamic effects, and vortices, we have always utilized bobs of streamlined shape, usually lenticular horizontal bobs. 
The suspension of our pendulum is a ring with a support base fixed in its upper interior, resting upon a ball that oscillates in a spherical cup. Refer to Fig.1.

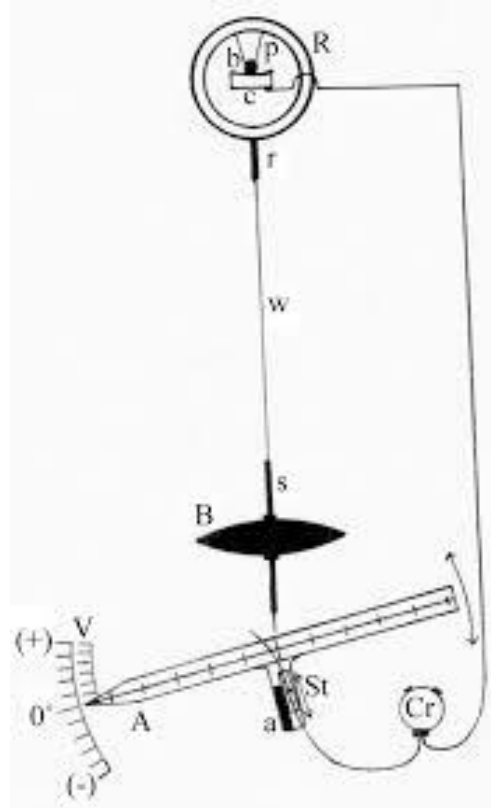

Figure 1. A sketch of an aerodynamic paraconical pendulum with chronometer and vernier.

For measuring the azimuth of the plane of oscillation, we utilize a vernier angular alidade having a precision of 0.1 sexagesimal degrees.

By convention, as in geodesy, we consider the azimuth to be positive if the rotation of the plane of oscillation of pendulum is clockwise and negative if it is anticlockwise.

For measuring the period of the pendulum, we employ an electronic chronometer with a precision of 0.001 second. A lead from a switch of the chronometer is connected to the suspension, and another lead is connected to a flexible contact wire. When the pointer of the pendulum touches the contact wire, the chronometer starts or stops.

After each release of the pendulum, we determine the mean average of the period several times, and thereby evaluate the damping of the oscillations. Then, using the average values of these period determinations after every release, we can construct a curve showing the variation of the period over the series of observations.

For measuring the proportion between the minor semi-axis and the major semi-axis of the ellipse of oscillation of the bob of the pendulum, a cross of millimetric paper is attached to the vernier alidade.

By our convention, the chirality of the motion is positive if the bob of the pendulum pursues a clockwise path around its ellipse, and negative if the bob pursues an anticlockwise path. In the following chirality graphs, we use +1 for positive, -1 for negative, and 0 when the oscillations are linear.

More detailed descriptions of our pendulum can be found in our previous article [4].

We also record the temperature, the atmospheric pressure, and the humidity.

\section{$3 \quad$ Experimental Results}

\subsection{Results Obtained During the Transit of Mercury of 9 November 2006}

The conjunction of the planet Mercury with the Sun of 8/9 November 2006 was in fact a transit. In Romanian Time $(\mathrm{UT}+2)$ this transit began in the evening of 8 November at $21 \mathrm{~h} 14 \mathrm{~m}$ and finished in the morning of 9 November at $2 \mathrm{~h} 10 \mathrm{~m}$. The maximum of the transit was on 8 November at $23 \mathrm{~h} 26 \mathrm{~m}$. At maximum, the ecliptic coordinates were $226^{\circ} 0^{\prime} 17.3^{\prime \prime}$ longitude, $0^{\circ} 0^{\prime} 7.3^{\prime \prime}$ latitude for the Sun, and $226^{\circ} 20^{\prime} 17.8^{\prime \prime}$ longitude, $-0^{\circ} 7^{\prime} 16.5^{\prime \prime}$ latitude for Mercury. The angular separation between Mercury and Sun was $7^{\prime} 3.2^{\prime \prime}$. 
This experiment was performed at Suceava Planetarium in the north of Romania, using a pendulum $3 \mathrm{~m}$ long installed in a small room, without windows, with thick walls, and having high thermal stability. For purposes of comparison, we began the experiment on 6 November at $18 \mathrm{~h} 45 \mathrm{~m}$ and finished on 10 November at $20 \mathrm{~h} 20 \mathrm{~m}$. During this interval, the pendulum was released nineteen times in five series of determinations.

After every release, six times, successively after 150 oscillations which corresponded roughly to 8 minutes 46 seconds, we stopped the chronometer, noted the time, once again started the chronometer, observed the azimuth of the plane of oscillation, the minor semi-axis b, and the major semi-axis a of the ellipse of oscillation, and noted the chirality of the pendulum motion. We now present the results obtained during this experiment.

\subsubsection{Results for the Variation of the Azimuth of the Plane of Oscillation}

In the Fig. 2 graphs, all azimuth values are shown. The differently marked lines show corresponding values at the same times after release, as follows:

$>$ Bottom line: the values after $8 \mathrm{~m} 46 \mathrm{~s}$

$>$ Next line upwards: the values after 2x8m46s

$>$ Next line upwards: the values after $3 \times 8 \mathrm{~m} 46 \mathrm{~s} . .$. and so on.

We can see that the values of the azimuth slowly increased to a maximum a little before the beginning of the transit, and declined sharply after the transit. In fact after the transit we even saw negative values, with the plane of oscillation of the pendulum having a tendency to rotate anticlockwise. Unfortunately we were not able to continue the observations further after the transit. Nevertheless, we consider that these graphs confirm that an Allais effect appeared during this conjunction.

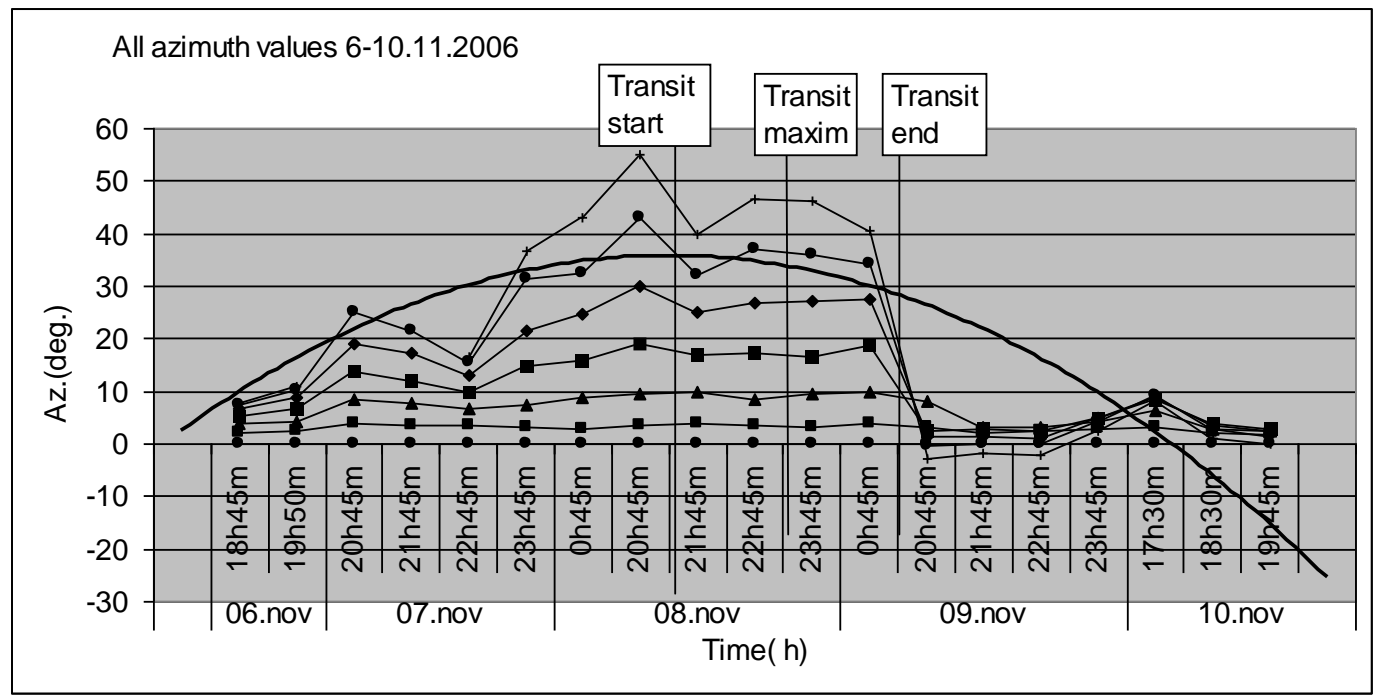

Figure 2. Variation of the azimuth of the plane of oscillation of the pendulum around the transit of Mercury of 8 November 2006

\subsubsection{Results for the Variation of the Proportion of the Semi-Axes of the Ellipse of Oscillation}

In the graphs of Fig. 3 we can see how the proportion $P=b / a$ between the semi-minor axis $b$ and the semi-major axis $a$ of the ellipse of oscillation of the bob of the pendulum varied.

Here we can see the same tendency: a slow increase of the proportion before and during the transit, and small values after the transit.

We conclude that the variation of the proportion $P$ is another criterion demonstrating that alignments of celestial bodies can cause anomalies in the behaviour of a pendulum. 


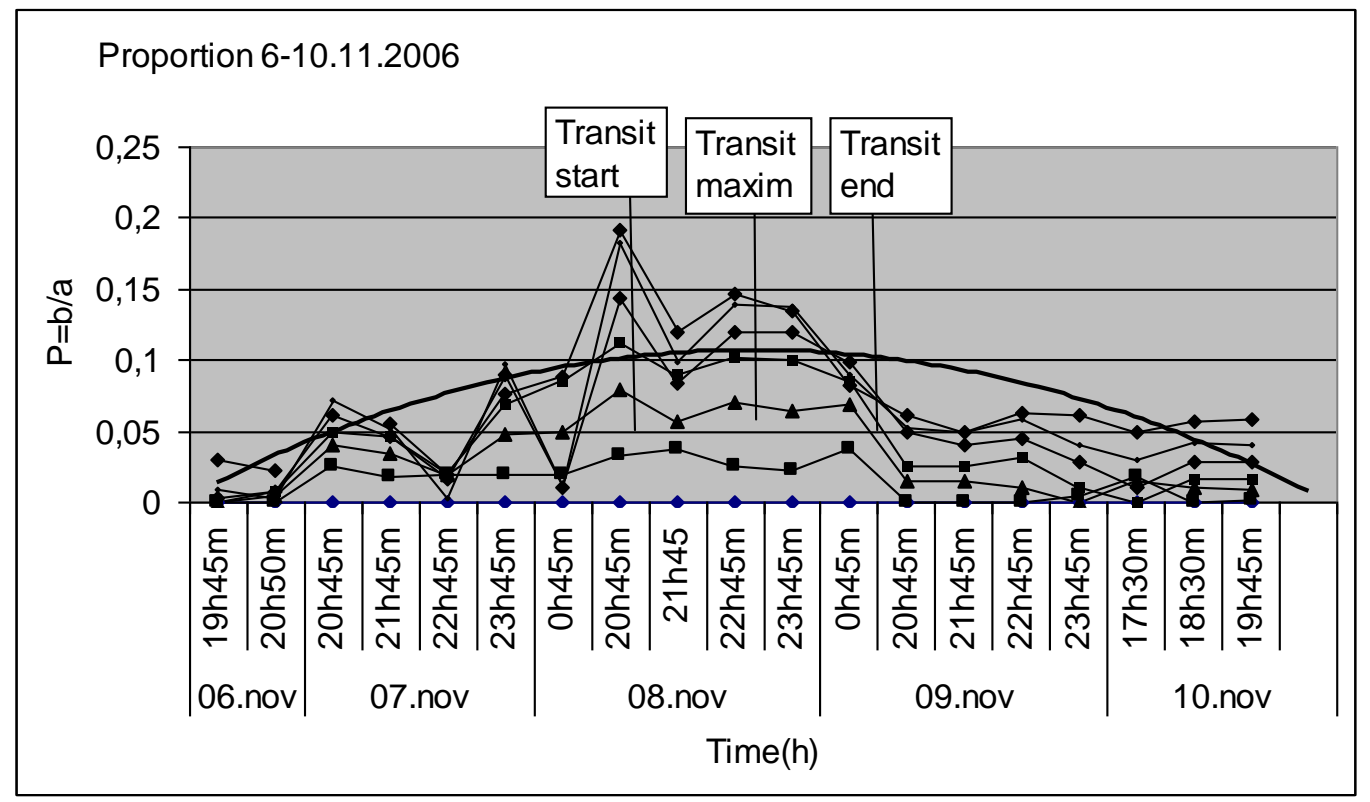

Figure 3. Variation of the proportion of the semi-axes of the ellipse of oscillation around the Mercury transit of 8 November 2006

\subsubsection{Results for the Variation of the Chirality of the Pendulum Motion}

In the Fig. 4 graph we present the variation of the chirality around the Mercury transit of 8 November 2006.

Analyzing the chirality after every $8 \mathrm{~m} 46 \mathrm{~s}$, we find that on the night of the transit the pendulum always described a clockwise path (positive chirality), while before and after the transit there were a number of anticlockwise cases (negative chirality).

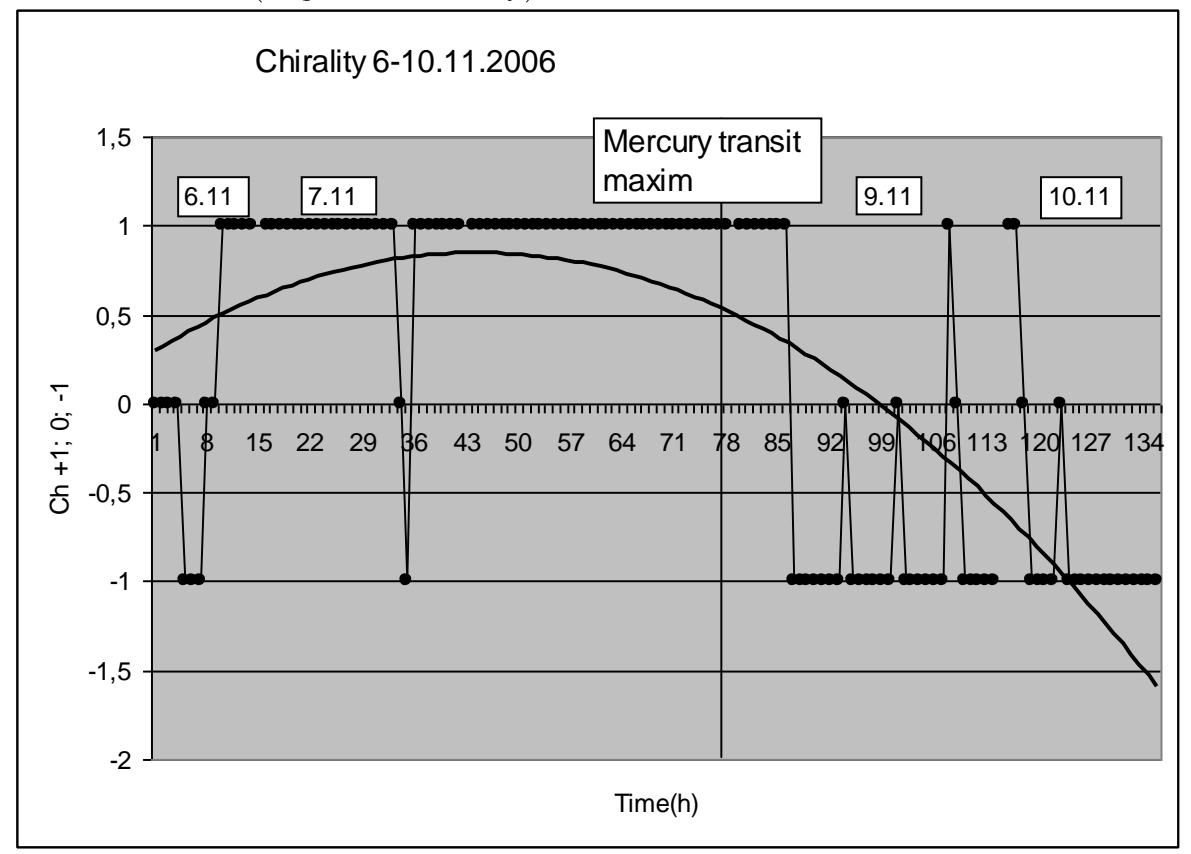

Figure 4. Variation of the chirality around the Mercury transit of 8 November 2006.

\subsubsection{Results for the Variation of the Period}

Fig. 5 gives graphs for the variation of the period during the Mercury transit of 8 November 2006 . 
During this experiment, the variation of the period of the pendulum did not exhibit any maximum during the transit; rather, we see a general tendency to reduction of the period. In other experiments we have also found such decreasing or increasing behaviour of the period over some days around a syzygy. This may be due to the influence of other celestial events. In this case, before the Mercury-Sun conjunction, the full Moon was on 6 November, a Mercury-Venus conjunction occurred on 7 November, and a Mercury-Mars conjunction occurred on 11 November. These three very close alignments may have acted as a single prolonged event. This situation of prolonged alignment appeared on other occasions.

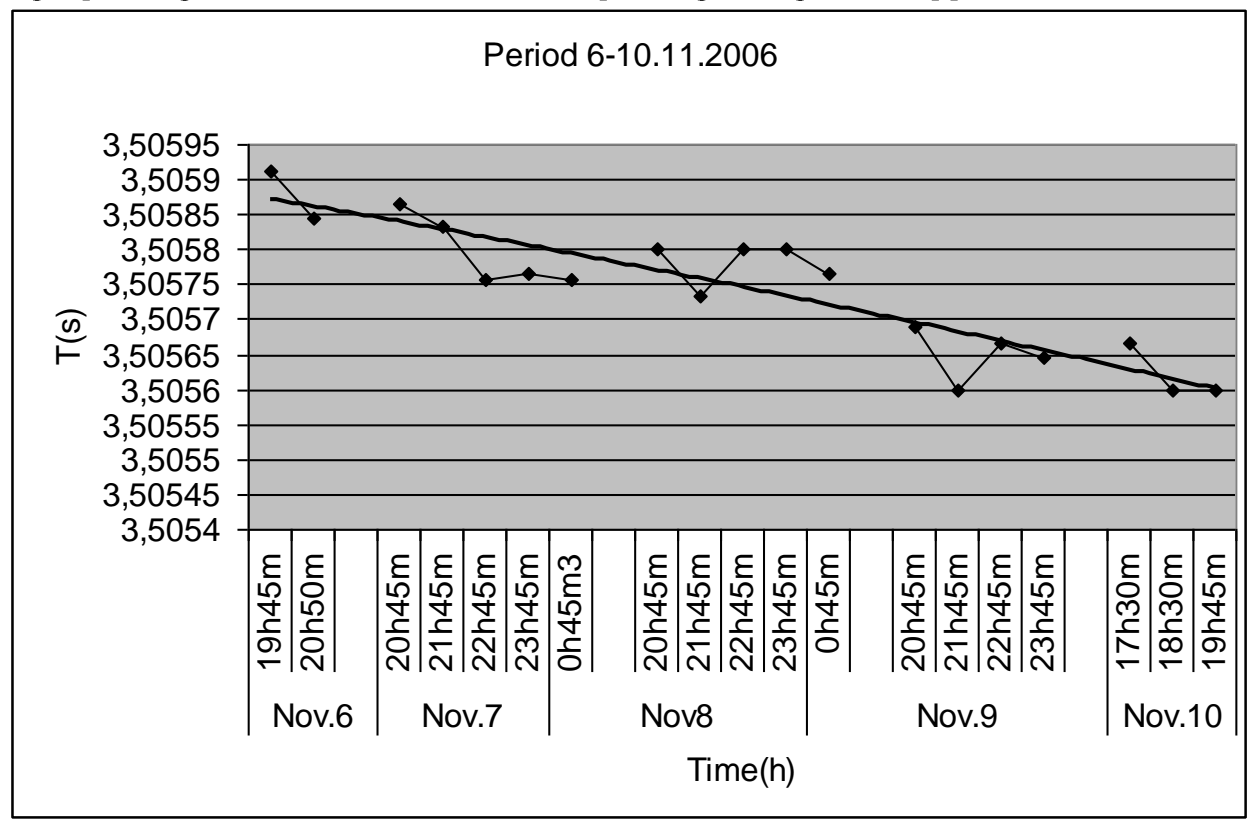

Figure 5. The variation of the pendulum period over 6-10 November 2006

\section{$3.2 \quad$ Results Obtained During the Mercury-Sun Conjunction of 14 July 2009}

On the occasion of the total solar eclipse of 22 July 2009 which passed across China, a team consisting of Professor Jinling Li, Chaoyuan Yang (a former astrophysicist at NASA), Botao Zhang (a student at the University of Nanjing), and the author, Dimitrie Olenici of Suceava Astronomical Observatory and Planetarium, performed a series of pendulum experiments over 4 to 24 July at the Astronomical Observatory in Shanghai, Sheshan Station, China. See Fig. 6.

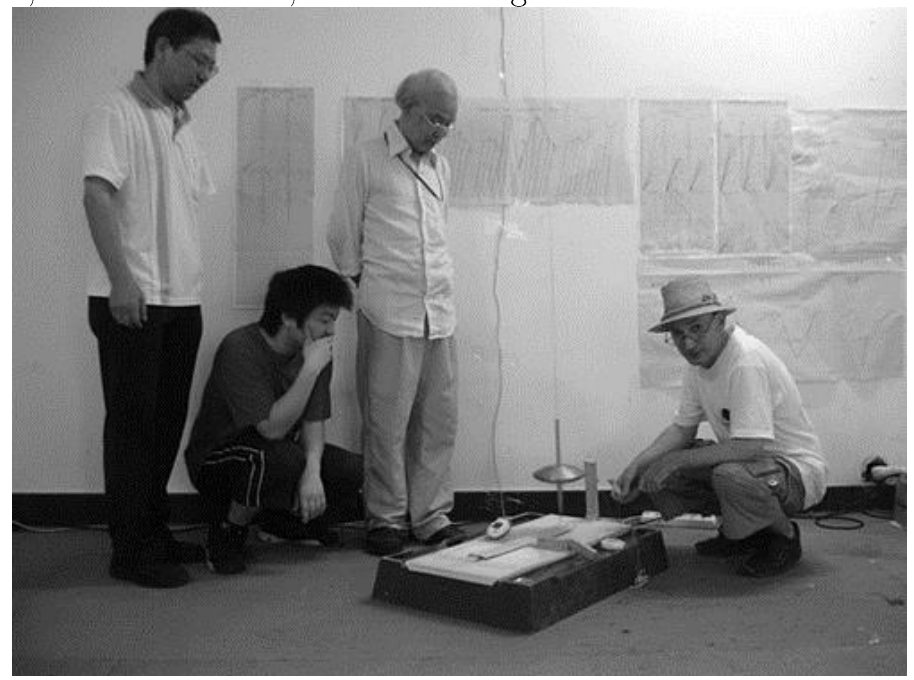

Figure 6. Jinling Li, Botao Zhang, Chao Yuan Yang, and Dimitrie Olenici performing a pendulum experiment at the Astronomical Observatory of Shanghai, Sheshan Station, during the total solar eclipse of 22 July 2009 (Photo by Adam Minter) 
During these experiments we utilized a pendulum of $2.4 \mathrm{~m}$ length with a bob of mass $9.0 \mathrm{~kg}$ that was shaped as a horizontal brass lens. The pendulum was released a total of 155 times. After every release, the azimuth of the plane of oscillation A, the major semi-axis a, the minor semi-axis b and the chirality Ch were observed five times, once every 140 oscillations (i.e. at intervals of 7 minutes 17 seconds).

In this marathon experiment the behaviour of the pendulum was studied during the following astronomical events: the lunar eclipse of July 7, the Jupiter-Neptune conjunction of July 9, the Mercury-Sun inferior conjunction of July 14, and the solar eclipse of July 22.

In this article we only present the results obtained during the Mercury-Sun conjunction of July 14.

In Chinese local time $(\mathrm{UT}+8)$, the Mercury-Sun conjunction occurred at $10 \mathrm{~h} 08 \mathrm{~m}$. The ecliptic coordinates of these two bodies were: $111^{\circ} 47^{\prime} 6.2^{\prime \prime}$ longitude and $-0^{\circ} 0^{\prime} 0.6^{\prime \prime}$ latitude for the Sun, and $111^{\mathrm{O}} 47^{\prime} 6.4^{\prime \prime}$ longitude and $1^{\mathrm{O}} 29^{\prime} 9^{\prime \prime}$ latitude for Mercury. The angular distance between Mercury and the Sun was 1029'19".

\subsubsection{Results for the Variation of the Azimuth}

The release azimuth of the pendulum was always in the direction $310^{\circ}$ northwest to $130^{\circ}$ southeast. The horizontal bottom line (round dots) corresponds to the pendulum release.

The Fig. 7 graph shows the values of the azimuth of the plane of oscillation on 13 July (the day before the conjunction), on 14 July (the day of the conjunction), and on 15 July (the day after the conjunction).

The lower curve (1) shows the values after 7 minutes 17 seconds.

The next curve (2) shows the values after 14 minutes 34 seconds.

The next curve (3)shows the values of the azimuth after 21 minutes 51 seconds, etc..

We can see very clearly that the precession of the azimuth assumed its smallest values exactly when the conjunction occurred. This demonstrates that an Allais effect was manifested.

The two maxima near minima during the conjunction are very interesting.

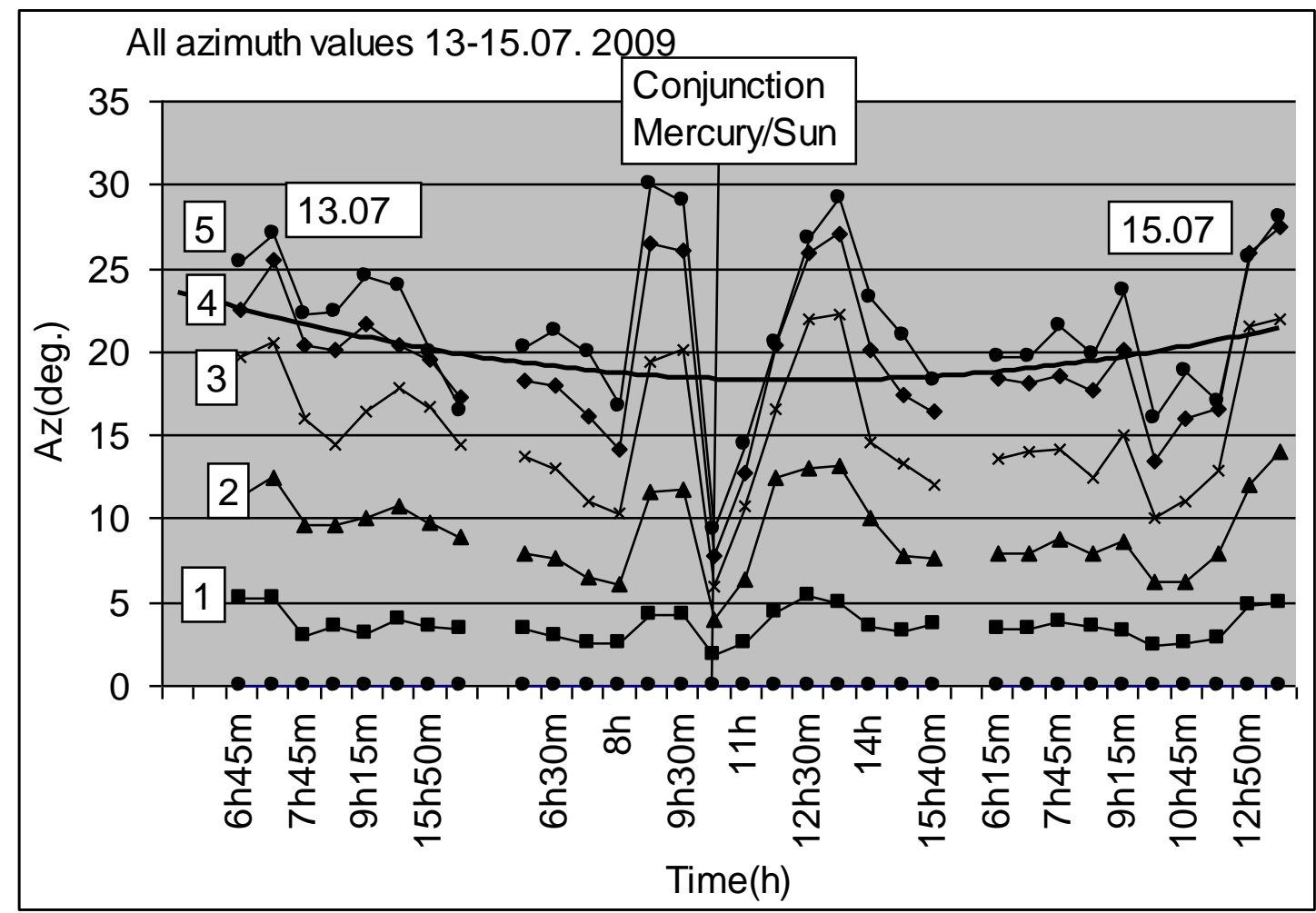

Figure 7. Variation of the azimuth of the pendulum oscillation plane around the Mercury transit of 9 July 2009 


\subsubsection{Results for the Variation of the Proportion of the Semi-Axes}

In the Fig. 8 graphs, we show the values of the proportion $P=b / a$ of the semi-axes of the ellipse of oscillation during this experiment around the Mercury transit of 14 July 2009.

The bottom straight line represents the proportion at the start.

Curve number 1 (square dots) represents the proportion after 7 minutes.

Curve number 2 (triangle dots) represents the proportion after 14 minutes.

Curves number 3 (cross dots) represents the proportion after 21 minutes.

Curve number 4 (diamond dots) represents the proportion after 28 minutes.

Curve number 5 (round dots) represents the proportion after 35 minutes.

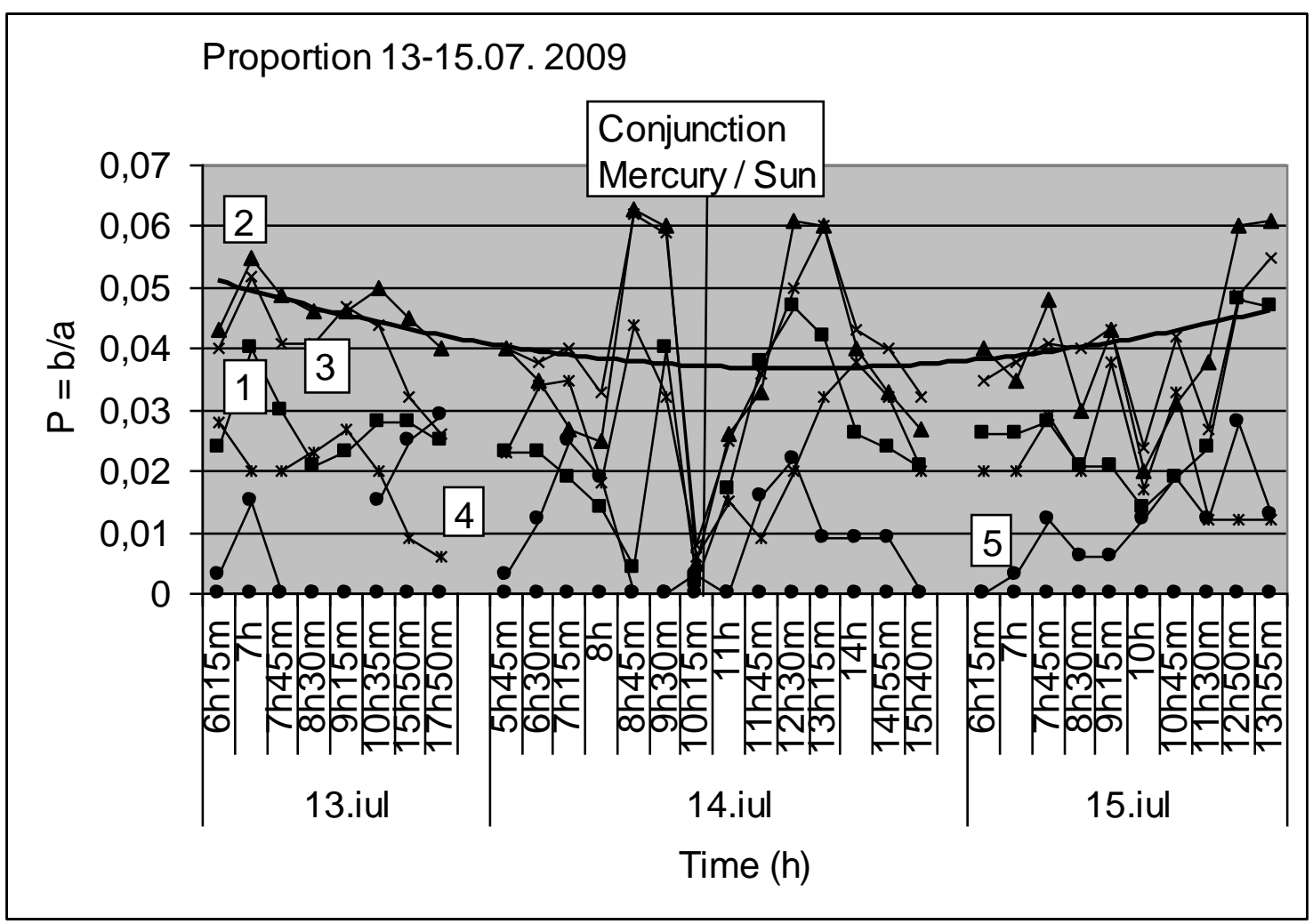

Figure 8. The values of the proportion of the semi-axes of the ellipse of oscillation of the pendulum around the Mercury conjunction of 14 July 2006

The amplitude at release, i.e. the initial semi-major axis a of the ellipse of oscillation, was always 230 $\mathrm{mm}$. Slowly this decreased over time to around $212 \mathrm{~mm}$ after $7 \mathrm{~m}, 196 \mathrm{~mm}$ after $14 \mathrm{~m}, 182 \mathrm{~mm}$ after $21 \mathrm{~m}$, $170 \mathrm{~mm}$ after $29 \mathrm{~m}$, and $160 \mathrm{~mm}$ after $36 \mathrm{~m}$.

The minor axis b had values between 0 and a maximum of $12.5 \mathrm{~mm}$.

For example:

$>$ On 14 July, at the beginning of observations at $8 \mathrm{~h} 45 \mathrm{~m}$, the values of the minor semi-axis were: 0 $\mathrm{mm}$ at start, $8.5 \mathrm{~mm}$ after $7 \mathrm{~m}, 12.5 \mathrm{~mm}$ after $14 \mathrm{~m}, 12 \mathrm{~mm}$ after $21 \mathrm{~m}, 7.5 \mathrm{~mm}$ after $29 \mathrm{~m}$, and 0 $\mathrm{mm}$ after $36 \mathrm{~m}$. The corresponding calculated values of the proportion $b / a$ were calculated as $0 / 0.04 / 0.063 / 0.062 / 0.044 / 0$.

$>$ On July 14 when the conjunction took place, after the start at $10 \mathrm{~h} 15 \mathrm{~m}$, the values of the minor semi-axis were $0 / 0.05 \mathrm{~mm} / 1 \mathrm{~mm} / 1.5 \mathrm{~mm} / 1 \mathrm{~mm} / 0.5 \mathrm{~mm}$, and the proportion had the respectively corresponding values 0/0.002/0.005/0.008/0.006/0.003.

Here we can see a similar situation as in the case of the azimuth curves: during the conjunction the values of the proportion were smallest, while it assumed very great values before and after the conjunction. Therefore, during this conjunction, the criterion of change of the semi-axis proportion was demonstrated. 
The fact is interesting that the proportion did not increase uniformly. In general the greatest values were 14 minutes and 21 minutes after the release (curves numbers 2 and 3)after that the proportion started to decrease (curves numbers 4 and 5 )

\subsubsection{Results for the Variation of the Chirality}

In the Fig. 9 graph we see that the greatest number of negative (anticlockwise) values of the chirality were on the days before and after the conjunction on 13 and 15 July, whereas, on the day of the conjunction, the pendulum only once revolved anticlockwise. This fact demonstrates that the chirality of the pendulum was dramatically affected during the Mercury-Sun conjunction of 14 July 2009.

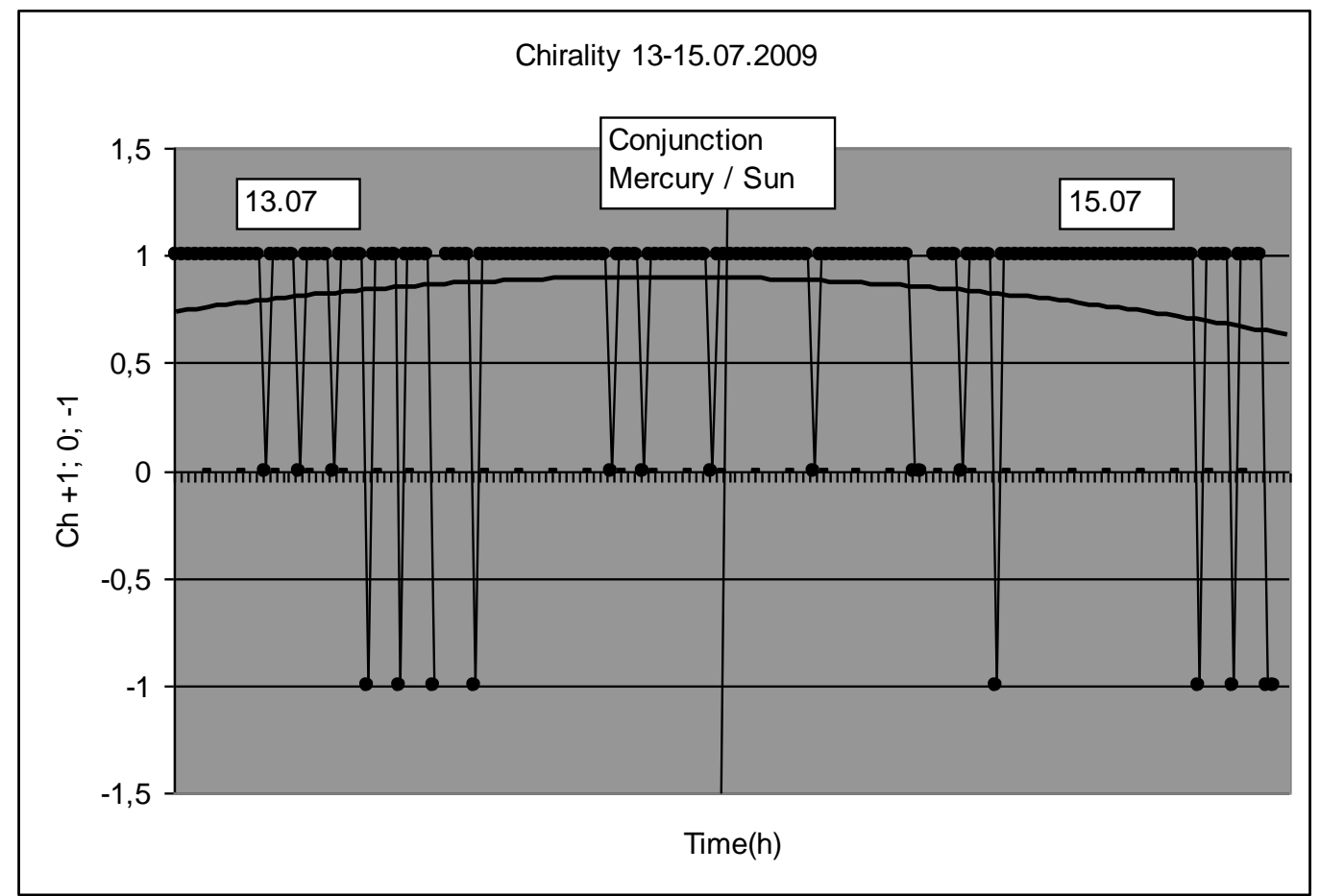

Figure 9. The change of chirality of the motion of the pendulum bob during the experiment of 13-15 July 2009 at Shanghai

\subsubsection{Results for the Variation of the Period}

In the Fig. 10 graph we show the variation of the average period of the pendulum after every release. For determining these values, after every release, five times in succession, we determined the average value after 140 oscillations had elapsed. Then we took the mean of these average values.

We see that on the days before and after the transit the period exhibited great fluctuations, while on the day of transit the curve ws smoother. Also the average values of the period were a little smaller on the day of transit, as compared with the values of the period on the days before and after conjunction, as follows: $T_{b}=3.118001 \mathrm{~s}$ on 13 July, $T_{c}=3.117969 \mathrm{~s}$ on the day of the conjunction, and $T_{a}=3.117976 \mathrm{~s}$ on 15 July after the conjunction.

We think that we can affirm that a Jeverdan-Rusu-Antonescu effect occurred.

Observation: In order for the length of the pendulum wire not to be affected by variations of temperature, we kept the temperature at the constant value of $20^{\circ} \mathrm{C}$ with air-conditioning. 


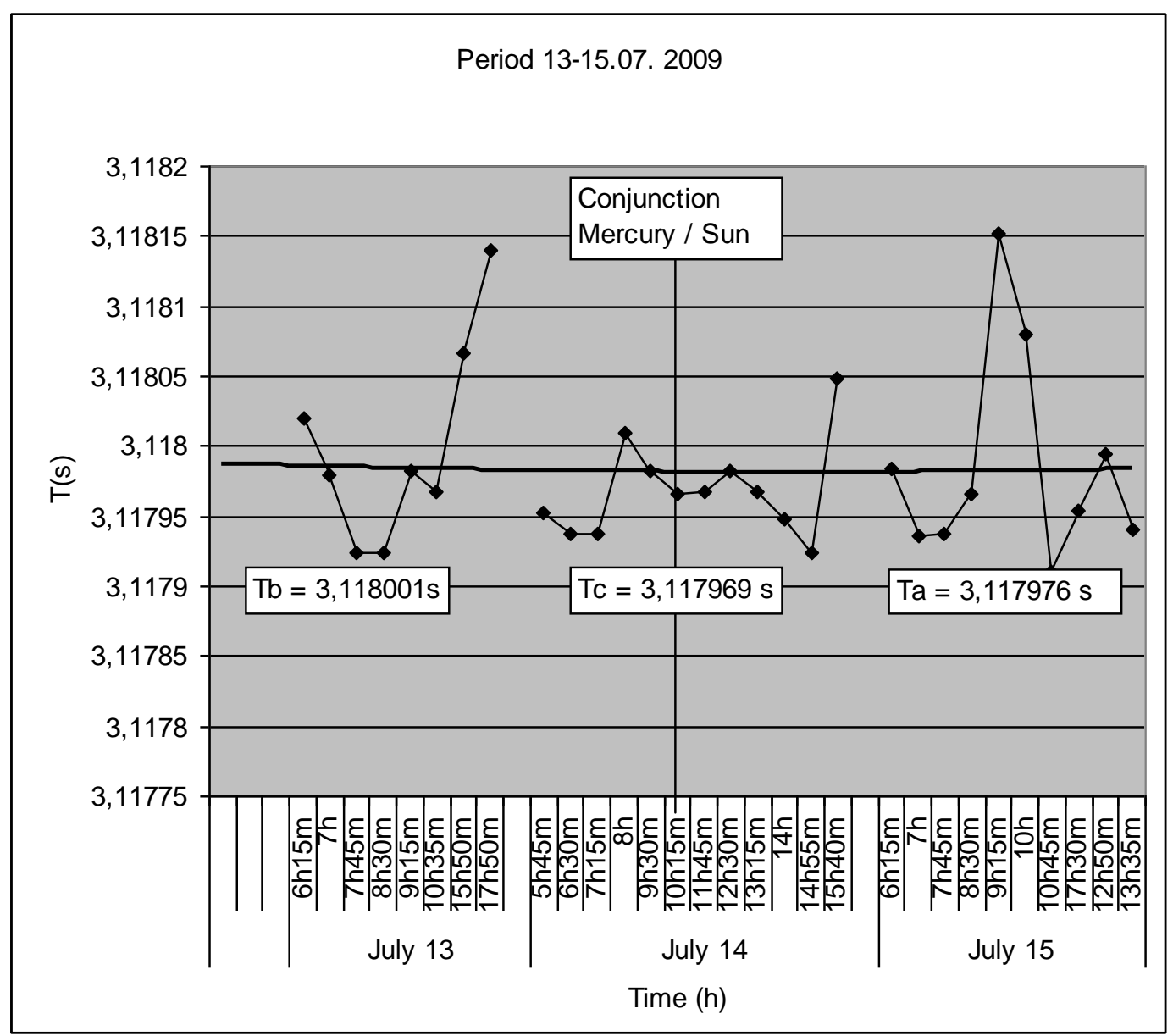

Figure 10. The variation of the pendulum period over 13-15 July 2009

\subsection{Results Obtained During the Occultation of Mercury by the Sun on 17 November 2015}

On 17 November 2015, an occultation of the planet Mercury by the Sun occurred. The angular separation was 15'. This event presented us with a challenge to do new pendulum experiments for comparison with the previous results during the transit and the conjunction of Mercury.

This time we performed experiments in a special building symbolically termed the "pendularium", constructed in the village Horodnic de Jos, Suceava county, Romania $\left(26^{\circ} 49^{\prime} 56^{\prime \prime} E\right.$ longitude, $47^{\circ} 51^{\prime} 39^{\prime \prime} \mathrm{N}$ latitude). In two towers incorporated into this building we have installed two pendulums with horizontal lenticular brass bobs having $24 \mathrm{~cm}$ diameter, $6 \mathrm{~cm}$ central thickness, and $13 \mathrm{~kg}$ mass. The lengths of both the pendulums are around $6 \mathrm{~m}$. Their alidades can be rotated so that, at release, the planes of oscillation of the pendulums can be parallel, or at right angles, or indeed along any desired directions.

\subsubsection{Results for the Variation of the Azimuth}

At Horodnic de Jos, in Romanian Time (UT +2$)$, the occultation began at $9 \mathrm{~h} 46 \mathrm{~m}$, reached maximum at $14 \mathrm{~h} 53 \mathrm{~m}$, and ended at $20 \mathrm{~h} 21 \mathrm{~m}$. In this experiment we utilised only one pendulum, which was released along the azimuth $335^{\circ}$ northwest - $145^{\circ}$ southeast at an initial amplitude of $500 \mathrm{~mm}$.

The observations were performed between $9 \mathrm{~h}$ on 16 November and $22 \mathrm{~h}$ on 18 November. During this interval, the pendulum was released 20 times. After every release, five times, at intervals of 6 minutes 44 seconds which corresponded to 83 oscillations, we recorded and restarted the chronometer and recorded the azimuth, the amplitude (i.e. the major semi-axis), and the minor semi-axis, and also noted the chirality. 
In the Fig. 11 graphs, we show the values corresponding to each release after every 6 minutes 44 seconds (the lowest curve gives the values after $6 \mathrm{~m} 44 \mathrm{~s}$, the next after $2 \times 6 \mathrm{~m} 44 \mathrm{~s}$, the next after $3 \times$ $6 \mathrm{~m} 44 \mathrm{~s}$, and so on).

We can see that the azimuth assumed great values before the occultation on 16 November and after the occultation on 18 November, while during the occultation on 17 November the azimuth took quite small values. The fact is very interesting that the azimuth decreased slowly before the maximum of the occultation, and increased sharply immediately after the end of the occultation.

These results enable us to claim that an Allais effect occurred during this occultation of Mercury by the Sun.

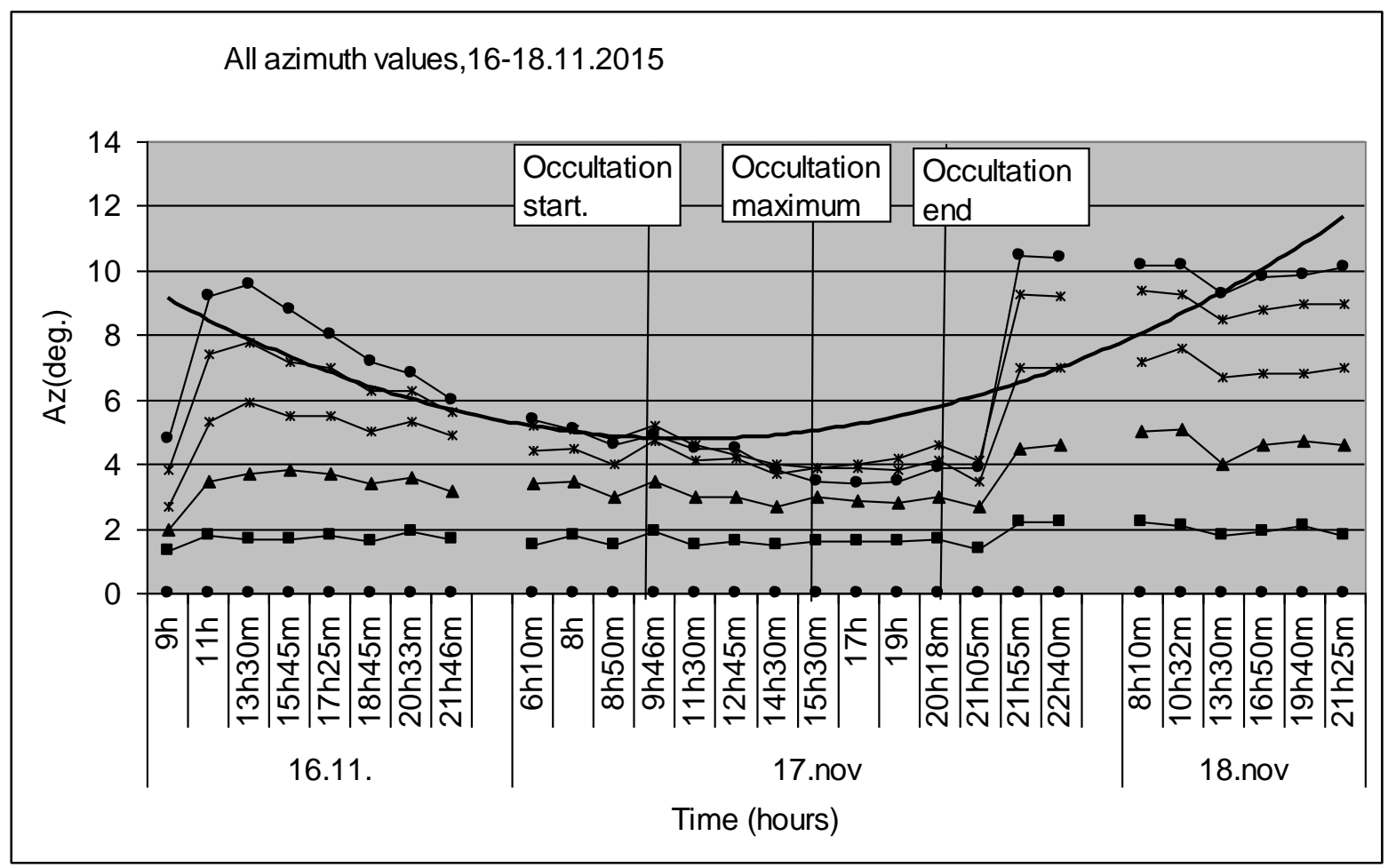

Figure 11. The variation of the azimuth of the plane of oscillation of the pendulum around the Mercury occultation of November 17, 2015

\subsubsection{Results for the Variation of the Proportion of the Semi-Axes}

The Fig. 12 graph shows the values of the proportion $P=b / a$ between the minor semi-axis $b$ and the major semi-axis $a$ of the ellipse of oscillation of the pendulum. In this case we see that, during the first 21 minutes after the release, the first, second, and third curves had small values before and during the occultation and began to increase after the occultation.

The readings at 28 and 35 minutes (the next two curves) had small values before the occultation, great values during the occultation, and again small values after the occultation.

These changes show that the criterion of change of the proportions of the ellipse of oscillation was satisfied. 


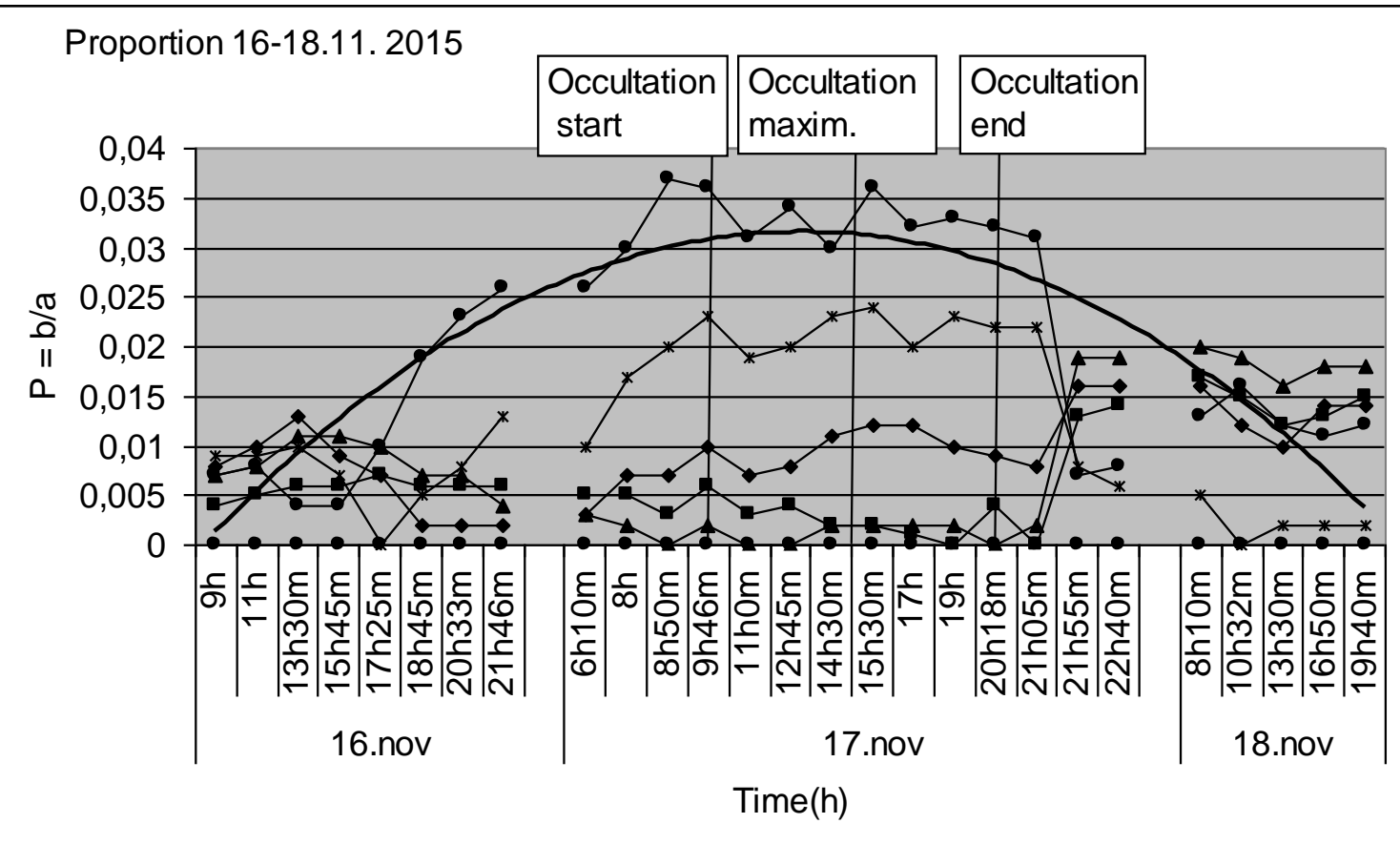

Figure 12. The variation of the proportions of the ellipse of oscillation of the pendulum during the Mercury occultation of 17 November 2015

\subsubsection{Results for the Variation of the Chirality}

From the Fig. 13 graph we see that on 16 November, the day before the occultation, and 18 November, the day after the occultation, there was a high proportion of positive chirality values (clockwise bob revolution).

However on 17 November, the day of the occultation, negative chirality values predominated. It appears that, on the day of occultation, in general the pendulum bob revolved anticlockwise. In our opinion, this significant change of chirality reveals an abnormality in the behaviour of pendulum during the occultation of Mercury by the Sun.

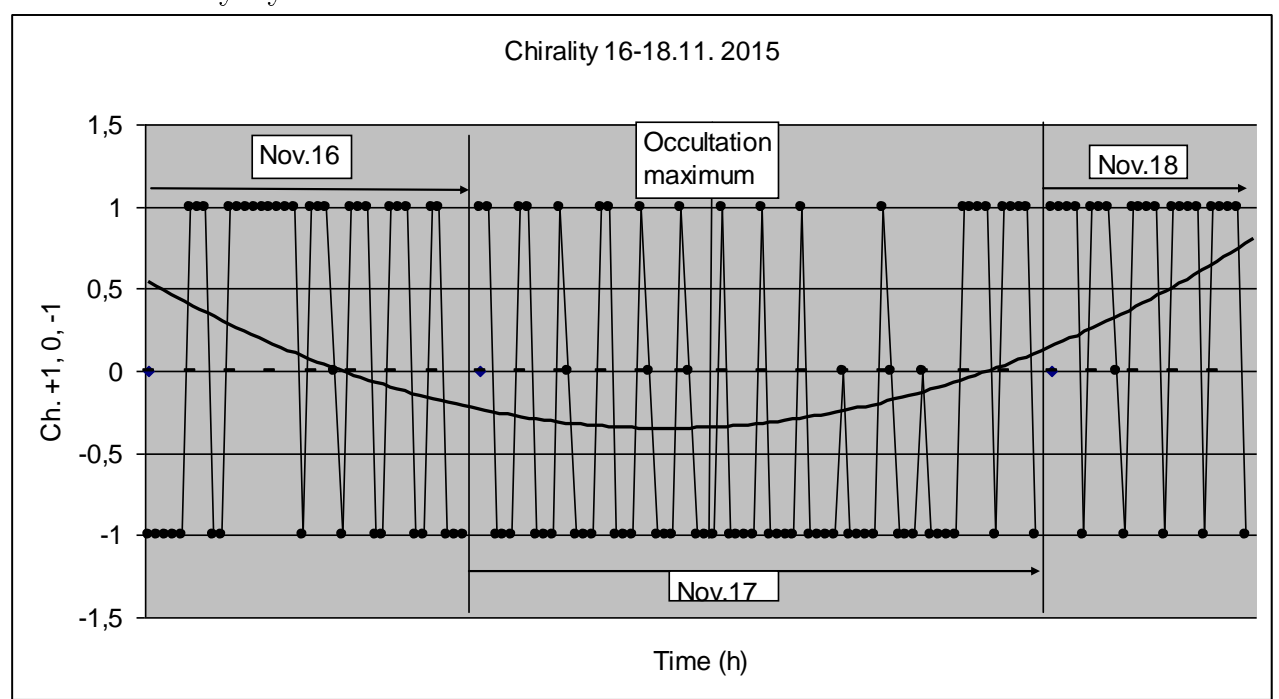

Figure 13. The variation of chirality during the Mercury occultation of 17 November 2015 


\subsubsection{Results for the Variation of the Period}

In view of the fact that even a small change of the pendulum length due to dilatation changes the value of the period, we monitored the pendularium temperature $\theta$. We placed an electronic thermometer near the base of the pendulum and a remote temperature sensor in the upper part of the tower near the pendulum suspension. Before every release, we noted the upper and lower temperatures and calculated their average value.

We also measured the pressure $P h$ (in millibars) and the humidity $H u$ (in percent). To fit these three parameters (temperature $\theta$, pressure $P h$, and humidity $H u$ ) on the same chart, we divided the pressure by 100 and the humidity by 10. Refer to Fig. 14 .

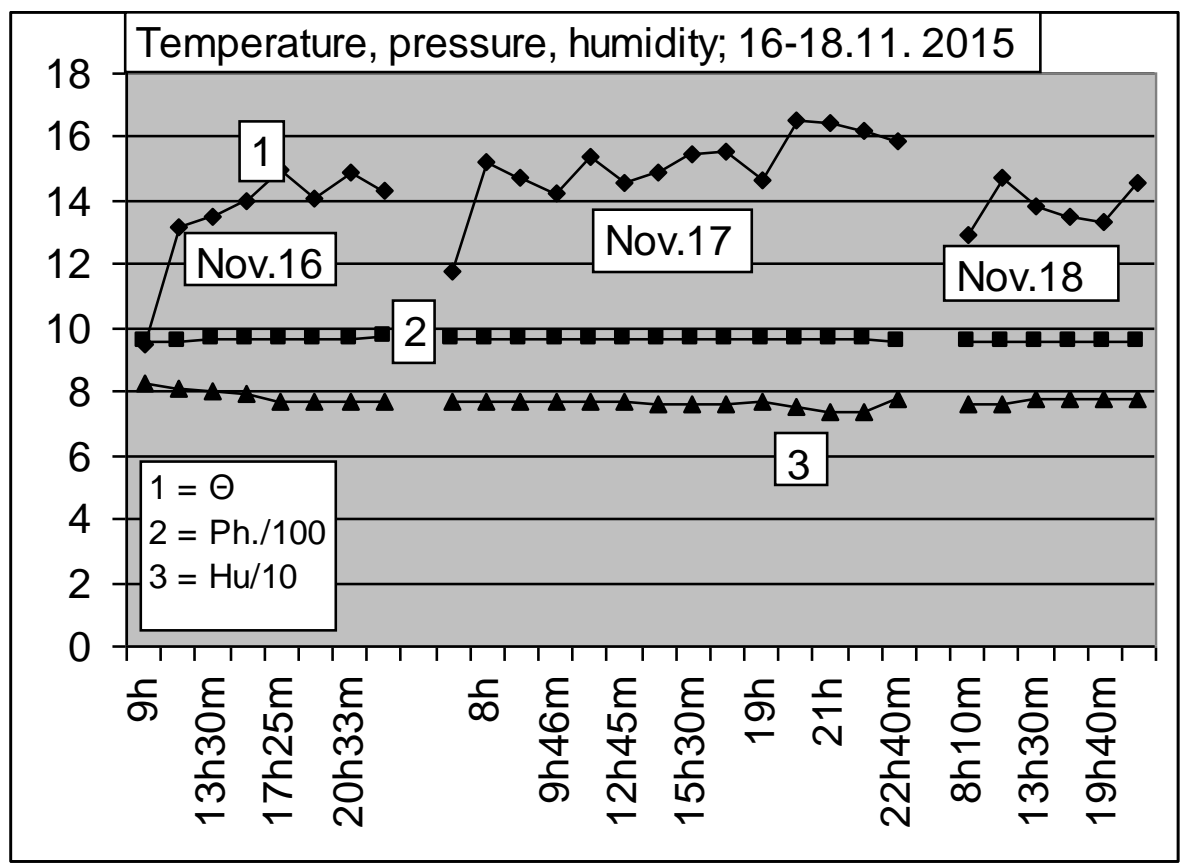

Figure 14. Graphs of temperature, pressure and humidity around the Mercury occultation of 17 November 2015

On the graph of Figure 15, curve number 1 represents the average values of the period measured after each release.

Comparing this curve on the graph of Fig. 14 with the temperature curve, we see that they are very similar, and we are at first sight impelled to believe that the variations of the temperature strongly influenced the variations of the period.

In order to investigate this matter further, we found the average value of all our temperature determinations over $16-18$ November, which was $13.825^{\circ} \mathrm{C}$. The closest temperature near this value was the value $13.85^{\circ} \mathrm{C}$. At this temperature the pendulum wire had length Lo (unknown), and the period To was experimentally measured to be $4.8500264 \mathrm{~s}$. We take this period $(4.8500264 \mathrm{~s})$ and this temperature $\left(13.85^{\circ} \mathrm{C}\right)$ as being a standard.

Taking in account the formula for the pendulum period and the law of dilatation, we can calculate the period of the pendulum $\mathrm{T}$ for any temperature $\Theta$ by using the formula

$$
T=T_{0} \sqrt{(1+\alpha \delta \Theta)}
$$

where $\alpha$ is the coefficient of thermal expansion of the wire and $\delta \Theta$ is the difference between the temperature $\Theta$ and the standard temperature $13.85^{\circ} \mathrm{C}$.

In our case, $\alpha=10 \times 10^{-6} \mathrm{~mm} / \mathrm{m}{ }^{\circ} \mathrm{C}$, and, by using the calculated values of temperatures, we obtain curve number 2 for the pendulum period. This curve is smoother, which shows that the variation of temperature did not dramatically influence the variation of period. Refer to Fig. 15. 
Period 16-18.11.2015

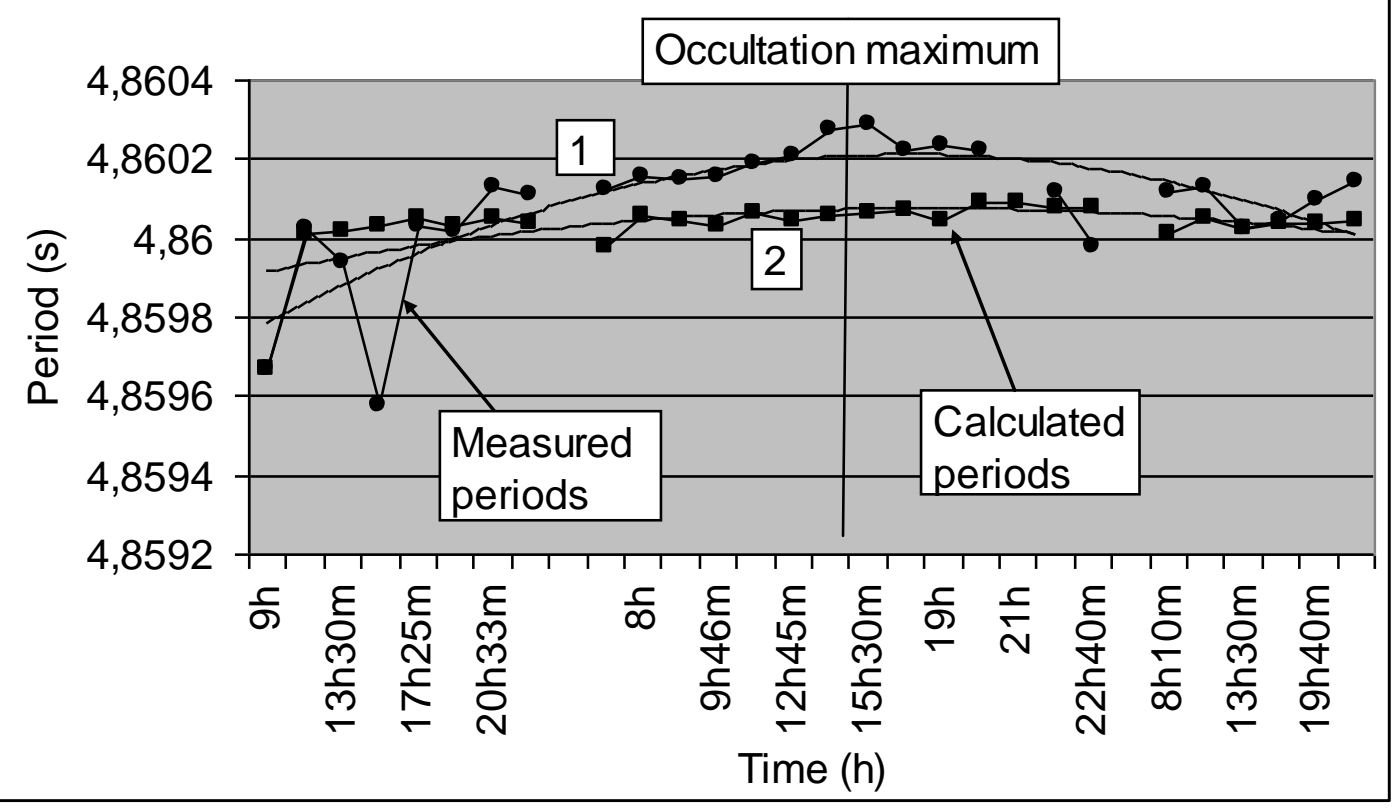

Figure 15. The pendulum period calculated as dependent upon the temperature (curve with square points) and the actually measured period (curve with round points) over 16-18 November 2015

Comparing the forms of the measured period curve and the calculated period curve, we find substantial difference: primarily, the values of the period seen around the maximum of the occultation are greater and have a tendency to appear convex. Curve number 2 does not present great fluctuations, has no maximum or minimum during the transit, and exhibits only a small convex tendency.

The greatest value of the calculated period was $T_{c}=4.860092 \mathrm{~s}$, which corresponds to a temperature increase of $2.6^{\circ} \mathrm{C}$.

The greatest period value that was measured during the maximum of the occultation was $T_{o c}=$ $4.8602868 \mathrm{~s}$, which would correspond to a quite unrealistic temperature increase of $8^{\circ} \mathrm{C}$.

The great difference between the calculated periods, which did not present great fluctuations around the transit, and the measured periods, which had very great values around the transit, confirms the presence of a Jeverdan-Rusu-Antonescu effect.

Observation: For the time being we reserve judgment to some extent, because we did not use any remote temperature sensors at the central part of the wire. In future, in order to calculate the dilatation of the wire much more precisely, we will utilise more temperature sensors.

\section{Conclusions}

1. A first conclusion is that these experiments confirm once again the fact that the Allais effect, the Jeverdan-Rusu-Antonescu effect, and other anomalies of the behaviour of a pendulum, initially observed during solar eclipses, have a more general character and also appear during conjunctions, transits and occultations of planets.

2. The anomalies observed during the transit of Mercury on 8 November 2006 confirm previous observations that such anomalies can appear even to an observer at a location where a planetary alignment is not optically visible. [5, 6]

3. The anomalies observed during these syzygies are similar to the anomalies observed during the Venus transits of 2006 and 2012 [7].

4. Many times we have observed a pronounced axial rotation of the pendulum around the maxima of eclipses, conjunctions, transits, and occultations. We think that this is the torsion effect discovered by A.F. Pugach using a very small torsion balance which he terms a torsind [8]. 
Our intention is, in future, to perform precise determinations of the effect of torsion of a paraconical pendulum, which may constitute a new type of pendulum anomaly.

In view of the fact that transits and occultations of planets are rarer events than eclipses, it is clear that, in order to monitor syzygy effects appearing during transits and occultations, it is necessary to perform observations persistently and patiently over long intervals.

For example these results could only be comparatively analyzed after three experiments over a period of nine years. The next such occasions in the following seven years will be: the Mercury transits on 9 May 2016 and 11 November 2019, the Mercury occultations on 4 May 2020 and 18 November 2022, the Venus occultation on 7 June 2016, the Mars occultation on 18 November 2023, the Jupiter occultation on 27 December 2019, and the Saturn occultation on 13 January 2020.

\section{Acknowledgements}

The author gives many thanks to the National Natural Science Foundation of China (Grant Nos. 10778635 and 10173019), the China Lunar Exploration Project (CE 1), the National 863 Program of China (Grants Nos. 2008AA12A209 and 2008AA12A210), and the Science \& Technology Committee of Shanghai Municipality (Grant No. 06DZ22101).

The author is grateful for the help from the staff of the Sheshan Station of the Shanghai Astronomical Observatory: Dr. Qing Lin, Mr. Jinawei Zhang, Mr. Minglong Zhu, Mr. Hongjian Pan, Mr.Juping Chen, etc.

The author gives many thanks to Prof. Jinling Li, astrophysicist Chao Yuan Yang, and student Botao Zhang for help and support.

Also the author acknowledges the enthusiastic cooperation of the Muzeul Bucovinei, Suceava and Stefan cel Mare University of Suceava, Romania.

Many thanks are also due to the Fondation Maurice Allais under guidance of the Fondation Paris Tech and to Mr. Guy Berthault of France, for financing the construction and the equipment of our pendularium in Horodnic de Jos.

Finally, the author thanks Thomas Goodey for revising the English text.

\section{References}

1. M. Allais, "L'Anisotropie de l'Espace", Paris, Clement Juglar, 1977.

2. G.T. Jeverdan, G.I. Rusu and V.I Antonescu, "Preliminary data on the behaviour of the Foucault pendulum during the solar eclipse of 15 February 1961" (in Romanian), An. Univ. Iasi Section I, 7, No. 2, (1961), 457.

3. D. Olenici, et al. "Short History of Our Research Into Allais and Jeverdan-Rusu-Antonescu Effects", in "Should the Laws of Gravitation be Reconsidered?", Hector A. Munera, editor, Apeiron, Montreal, 2011, 207-221.

4. D. Olenici, et al. "Syzygy Effects Studies Performed Simultaneously with Foucault Pendulums and Torsinds during the Solar Eclipses of 13 November 2012 and 10 May 2013", International Journal of Astronomy and Astrophysics, 2014, 4, 39-53.

5. D. Olenici, "The Sarawak antieclipse pendulum experiment", Anuarul Complexului Muzeal Bucovina, XXXIVXXXV-XXXVI, Volume 2007-2008-2009, Suceava, 2009.

6. D. Olenici and A.F. Pugach, "Precise Underground Observations of the Partial Solar eclipse of 1 June 2011 Using a Foucault Pendulum and a very Light Torsion Balance", International Journal of Astronomy and Astrophyscs, 2012, 2, 204-209.

7. D. Olenici et al., "Studies on syzygy effects with aerodynamic paraconical pendulum during the transits of Venus of June 8, 2004 and June 6, 2012", Journal of Advanced Research in Physics, Vol.5, No1, (2015)

8. A.F.Pugach, "The Torsind - A Device Based on a New Principle for Non-Conventional Astronomical Observations", International Journal of Astronomy and Astrophysics, 2013, 3, 33-38

9. Internet

NASA-SKYCAL Sky Events Calculator

Calsky: Astronomical and Space Calendar

Cybersky 